Revista Española de Antropología Americana ISSN: 0556-6533

http://dx.doi.org/10.5209/reaa.70442

\title{
Conectando con la Amazonia transparente. El cuerpo afroindígena y la comunicación más allá de las élites
}

\author{
Aníbal G. Arregui ${ }^{1}$
}

Recibido: 2 de julio de 2020 / Aceptado 16 de agosto de 2020

Resumen. En Brasil, el término quilombola -o afroindígena en algunas corrientes antropológicas- designa descendientes de esclavos africanos que huyeron y formaron comunidades en Amazonia y otros lugares aislados. Circunvalando los parámetros puramente etnohistóricos del "quilombolismo", en este ensayo sugiero repensar las formas locales de identificación y comunicación política a través de la etnografía del cuerpo. Me baso en el trabajo de campo realizado entre 2006 y 2019 en el río Erepecurú para visibilizar el cuerpo como un medio de comunicación afroindígena que, si bien está ahí, aparece como una entidad transparente en buena parte de la literatura militante sobre la Amazonia negra. Desde esa Amazonia menos escuchada, argumentaré que el cuerpo afroindígena no sólo materializa un eficaz medio de comunicación local, sino que también recrea una colectividad sociopolítica a la sombra de las élites locales, institucionales e intelectuales.

Palabras clave: Amazonia; quilombolas; cuerpo; élites; comunicación; transparencia.

\section{[en] Connecting with Transparent Amazonia. The Afroindigenous Body and the Communication beyond Elites}

\begin{abstract}
In Brazil, the term quilombola -or afroindigenous peoples in certain anthropological traditions- designates the descendants of runaway African slaves who formed communities in Amazonia and other isolated places. Circumventing the ethno-historical parameters of "quilombolismo", in this essay I suggest rethinking local forms of political identification and communication through an ethnography of the body. I draw on fieldwork conducted between 2006 and 2019 in the Erepecurú River (Pará, Brazil) to render visible the afroindigenous body as a medium which might be there, but appears as a transparent entity in most part of militant literature on black Amazonia. From this less visible Amazonia, I will argue that the afroindigenous body materialises an efficient mean of communication and recreates a sociopolitical collectivity underneath local, institutional and intellectual elites.
\end{abstract}

Keywords: Amazonia; quilombolas; body; elites; communication; transparency.

Sumario. 1. Introducción. 2. Kinestesias de lo político. 3. Corporeizando la historia del quilombolismo. 4. Comunicación desde la (falsa) transparencia etnográfica 5. Conclusión. Auto-representación y la vida digital de los cuerpos. 6. Referencias.

Cómo citar: Arregui, Aníbal G. 2020. "Conectando con la Amazonia transparente. El cuerpo afroindígena y la comunicación más allá de las élites". Revista Española de Antropología Americana 50: 291-306.

1 Universidad de Barcelona. anibal_garcia_arregui@ub.edu 


\section{Introducción}

Cuando llegué por última vez a la comunidad quilombola de Varre Vento, una tarde de febrero de 2019, tres niños se habían perdido en la selva durante una batida de caza. El manto de nubes oscuras se espesó al atardecer. Algunos adultos se adentraron en el bosque para localizar a los pequeños, de 8,9 y 12 años, cuando la lluvia ya arreciaba y la noche hacia presencia. No los encontraron. Tuve la sensación de ser el único que no logró conciliar el sueño aquella noche. Conocía a los niños de mis anteriores visitas. A pesar de todo, la gente en la comunidad reaccionó con una tranquilidad que en aquel momento parecía incomprensible. Y se durmieron.

Con la primera luz del alba llegó una canoa descendiendo el río. El pescador informó de que los tres niños habían pasado la noche en la cabaña de un familiar, a la que llegaron ya entrada la noche. Prefirieron no regresar a la comunidad en medio de la tormenta. Uno de los cazadores que sí habían vuelto la noche anterior dijo con gesto confirmatorio: "fue aquel golpe de sapupema". Se refería al golpeo del dorso de un machete en la reverberante raíz tubular de un árbol llamado samaumeira, un modo tradicional de comunicación entre cazadores. El hombre había escuchado como los niños daban la señal de su localización aproximada. Podría haber sido una señal de socorro, pero como provenía de las inmediaciones de una cabaña, el sonido del golpeo fue interpretado como un mensaje de llegada a lugar seguro. Pregunté si no les había quedado la duda de qué significó aquel "golpeo de sapupema" en medio de la noche y la tormenta. El padre de uno de los niños ladeó la cabeza hacia un lado, como contemplando esa posibilidad, para luego afirmar que "los quilombolas no siempre necesitan palabras para decir cosas".

En las comunidades afrodescendientes del bajo Amazonas, la comunicación no verbal-corporal es un crucial articulador de las relaciones cotidianas y, por lo tanto, un importante aspecto de la identidad local. Los grupos afrodescendientes de la región se conocen como quilombolas en las narrativas más comunes, y como afroindígenas en algunas corrientes antropológicas especializadas (Goldman 2014). En este ensayo me aproximo a la comunicación corporal afroindígena en el río Eprepecurú (Pará, Brasil) con el propósito de visibilizar y deshacer la transparencia con que se trata esa forma de relación-comunicación desde perspectivas analíticas centradas en la exhortación verbal de la identidad, tanto la identidad afro como la indígena. Una de las características de la comunicación en la selva es que las noticias circulan entre personas y comunidades con sorprendente rapidez y sin necesidad de una interface tecnológica como la radio o el teléfono. En el río Erepecurú el medio selvático es a la vez la materialidad o canal que media los mensajes. En otras palabras: el medio ecológico es también un ubicuo y eficiente medio de comunicación. En ello juegan un papel crucial los cuerpos y su capacidad para emitir signos y transmitir mensajes no necesariamente verbales. Los cuerpos son parte constitutiva de un sistema inter-comunicado por personas que reman, caminan largas distancias, comparten comidas, intercambian historias u objetos, o simplemente se echan una mano en tareas domésticas de reparación o aprovisionamiento. Esa comunicación inter-corporal no solo materializa un eficaz sistema de transmisión de mensajes, sino que también recrea una colectividad sociopolítica a la sombra del discurso identitario de las élites locales, intelectuales e institucionales.

En Brasil, el llamado "movimiento quilombola" describe una corriente política que reivindica de manera explícita la ciudadanía basada en la ancestralidad africa- 
na y los derechos territoriales adquiridos como remanentes de esclavos cimarrones (Almeida 2009; Andrade 1998). De manera alternativa, en este ensayo me aproximo al "movimiento quilombola" en un sentido literal-cinético de la expresión. Con ello, parto de una versión del "quilombolismo" inspirada por la etnografía de unas formas corporales específicas de moverse y comunicarse. Desde una propuesta alternativa pero potencialmente complementaria a la historiografía de la región, el argumento circunvala las representaciones etnohistóricas acerca de quiénes son los quilombolas en relación directa y explícita a su pasado. Lo que busco no es tanto una crítica de la historiografía como una apertura metodológica con respecto a algunos discursos de exclusivo énfasis etnohistórico que son compartidos por ONGs y representantes políticos, tanto de la esfera local como de ámbito nacional. En concreto, sugiero una alternativa analítica que pueda articularse con esos enfoques que crucialmente han resaltado la "instrumentalidad jurídica" de las reivindicaciones étnicas de los afrodescedientes en Brasil (ver Arruti 2002; Boyer 2002, 2011; Larrea y Ruiz-Peinado 2004; Farfán 2015). De hecho, este es precisamente un momento social crítico en Brasil, un episodio de la historia que pone en entredicho las conciliaciones que trajo la Constitución de 1988, y que reclama cierta creatividad por parte de la Antropología para reactivar el quilombolismo de manera innovadora y políticamente efectiva (Fagundes 2020).

Un primer punto de partida es la re-denominación del colectivo que protagoniza este texto. Si bien es necesario reconocer la reivindicación por parte de algunos de mis interlocutores de su identidad quilombola, es frecuente que muchos moradores de las comunidades del río Erepecurú se auto-definan como ribeirinhos, en referencia a su pasado de mestizaje amerindio-africano-europeo (ver Nugent y Harris 2004; Arregui 2013, 2014, 2018). La identidad etnohistórica es un asunto complejo y altamente politizado en Amazonia por sus consecuencias en la titularidad de territorios habitados por poblaciones nativas (ver abajo). Los criterios intelectuales y jurídicos más aceptados delimitan la identidad quilombola en base una ancestralidad africana, el pasado de esclavitud, y la épica del cimarronaje. Sin embargo, en algunos círculos especializados de la antropología brasileña se emplea la idea de afroindígena para resaltar no solo las numerosas generaciones afro que han ya poblado la selva tropical americana, sino también los paralelismos cosmológicos, intercambios económicos y sinergias ecopolíticas que siempre ha habido entre quilombolas y las poblaciones amerindias de la Amazonia (Goldman 2014; Sauma 2014).

Mi propuesta de una etnografía corporal ${ }^{2}$ de la comunicación afroindígena surge en parte de una circunstancia azarosa. Entre los años 2006 y 2019 realicé la mayor parte del trabajo de campo en una comunidad quilombola llamada Varre Vento (río Erepecurú, Pará, Brasil) en la cual no hay representantes de las élites políticas locales. Estas élites son descendientes directos de los antiguos sakaka o chamanes, muchos de los cuales ahora ostentan cargos de representación política y organizan su vida entre las comunidades de la selva y el núcleo urbano de Oriximiná, situado a unas 10 horas de barco. De manera general, el entorno familiar de estos antiguos

\footnotetext{
Me refiero a una etnografía con foco en las prácticas físicas observables y los afectos y sensaciones que pueden asociarse a esas prácticas desde la perspectiva de mis interlocutores. La propuesta conecta así con lo que en inglés se conocen como teorías del embodiment, cuyas diversas traducciones al castellano -incorporar, encarnar, encorporar, corporear-contienen importantes matices teóricos y analíticos, tal y como han mostrado Olatz González-Abrisketa y Carlos García-Grados (2018)
} 
sakaka, que viven en tres de las comunidades más pobladas de la región, ha ido asumiendo los discursos políticos del "movimiento quilombola", la mediación con investigadores de campo y representantes institucionales, y la gestión de recursos económicos e insumos tecnológicos que vienen de fuera.

Como ilustraré más abajo, el hecho de alojarme en Varre Vento y establecer lazos de confianza en una comunidad donde mis interlocutores no eran parte de la élite político-chamánica local, me llevó a realizar una etnografía en la que discursos militantes acerca de los quilombolas solo tenían un papel secundario. Por un lado, este hecho me dio acceso a una realidad etnográfica diversa y "multivocal" -en su diversidad de intereses ecopolíticos- de los grupos afroindígenas locales y sus medios (Orobitg y Canals, 2020). Por otro lado, la distancia con respecto a esas representaciones y medios más visibles me llevó, de manera no premeditada, a una delineación "multimodal" (ibíd.) del campo de estudio en la que prácticas de horticultura, caza y pesca, y las percepciones sensoriales de esas prácticas, pasaron a un primer plano de la investigación etnográfica. Estas actividades resultaron ser un aspecto práctico y central de la cultura quilombola que mis interlocutores reivindicaban en el día a día. Por así decirlo, me vi involucrado en un contexto de actividades cotidianas, sensaciones e interacciones que la literatura regional trata como un aspecto transparente, es decir, algo que está ahí, pero sólo como antesala de la verdadera substancia de un quilombolismo que generalmente es entendido como un discurso histórico y político.

En ausencia de esos discursos homologados por intelectuales y activistas acerca de la identidad de los quilombolas, mis primeras y más duraderas percepciones sobre las formas de relación y comunicación locales surgieron de los contrastes que ellos mismos establecieron con respecto a un visitante, en este caso el que escribe. Como expongo más abajo, entre esos contrastes figura la identificación de un diferente jeito corporal o forma de hacer en mis a menudo infructuosos intentos de observación participante en diversas actividades, así como en mi obsesivo uso de medios -cuaderno de notas, la cámara, grabadora- a los que mis interlocutores encontraban un sentido más lúdico que práctico.

El resto del ensayo presenta esta etnografía de la comunicación corporal afroindígena en cuatro secciones. Primero evoco la importancia del jeito corporal, o forma de hacer y comunicar quilombola, y sus correspondientes propiocepciones o kinestesias. Me aproximo a la auto-percepción -y autoafirmación- de la rutina diaria como expresión distintiva del quilombolismo, proponiendo una identificación colectiva y corporeizada que puede aportar tanto una alternativa como un complemento a las habituales representaciones etnohistóricas. En la siguiente sección muestro dos giros analíticos que posibilita la evocación etnográfica de la comunicación corporal: por un lado capto y visibilizo formas de comunicación no verbal que son de crucial importancia en la vida diaria y la identidad en el Erepecurú. Por otro lado, sugiero que la atención al cuerpo deshace la falsa transparencia de los representantes (Spivak 1988), de manera que permite ubicar la posición discursiva, y parcialmente externa, de las élites locales, políticas e intelectuales, incluyendo aquí a las élites etnográficas. La última sección plantea las diferentes implicaciones que tienen la comunicación encarnada y la comunicación digital para el ejercicio de auto-representación (Orobitg et al. 2021). En diálogo con el resto de contribuciones al presente número, propongo indagar en las posibilidades de mediación intelectual que nos brinda el considerar los cuerpos y sus movimientos como legítimos medios de comunicación afroindígena. 


\section{Kinestesias de lo político}

En la Amazonia afroindígena, las prácticas diarias de caza, pesca, horticultura, recolección o incluso el ocio - fútbol, baile, baños en el río- conforman una constelación de "técnicas corporales" (Mauss 1968) que orientan no solo aspectos tecnológicos de la producción, sino también formas locales de relación entre personas y de interacción con el entorno (Arregui 2013, 2015; Scaramuzzi 2015). Esa constelación de habilidades se designa localmente como jeito: forma de hacer. En este contexto, la noción de jeito describe un modo de actuar corporalmente que responde a un particular "aprendi-

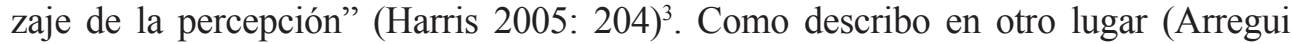
2020), el jeito articula de manera clave la identidad individual y la pertenencia a un colectivo: por un lado, señala una inteligencia práctica local que permite a los individuos coordinarse de manera flexible y eficiente en las tareas diarias. Por otro lado, el jeito encuadra una corporalidad distintiva, una suerte de habitus afroindígena sobre el que no sólo se sostiene la estrategia productiva sino también un sentido somático de pertenencia (Figura 1).
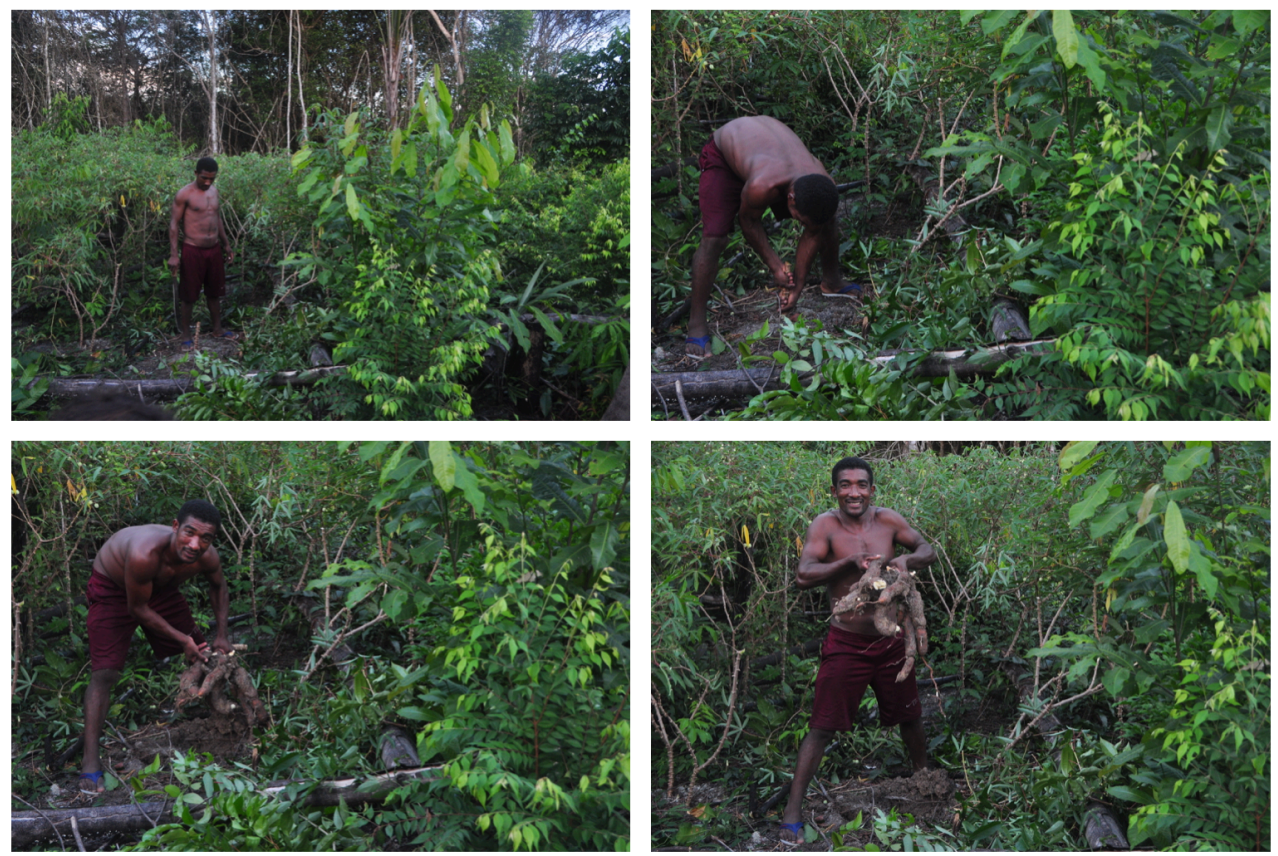

Figura 1. La extracción de tubérculos de mandioca como parte del jeito o corporalidad local (fotografía del autor).

El jeito no se comunica verbalmente, sino que se transmite por observación e imitación. Lo más frecuente es que aquellos que gozan de mayor experiencia prác-

La idea de jeito tiene un campo semántico más amplio en el contexto del portugués-brasileño. De manera más general el concepto no se refiere tanto a una característica corporal como a "un modo rápido y eficiente de alcanzar un objetivo saltándose una norma universal y usando en su lugar los recursos sociales y personales propios" (Barbosa 1995: 36). 
tica señalen al neófito la necesidad de "aprender mirando" (aprender olhando) o simplemente "haciendo" (fazendo), esto es, acompañando de manera mimética una actividad hasta que se adquiere una sensación kinestésica de la tarea. Aquí propongo observar etnográficamente el jeito afroindígena como "la punta de iceberg" de un conocimiento tecnológico-corporal complejo (Pfaffenberger 1992: 508), cuya principal vía de transmisión no es la palabra, sino la imitación y la práctica (Bloch 1998; Downey 2010; Arregui 2014).

Para ser aprendido, el jeito debe ser observado, practicado y experimentado en el propio movimiento corporal. La puesta en práctica del jeito va así acompañada de una "sensación kinestésica", es decir, de la "percepción del propio movimiento (kinesis)" y, de manera más general, de "experiencias propioceptivas que incluyen sensaciones musculares, experiencia del esfuerzo, fuerza, equilibrio, etc." (Moran y Cohen 2012: 180-181). El fenómeno perceptivo de las kinestesias ha sido ampliamente estudiado desde la psicología y la fenomenología, pero contiene aspectos de potencial interés para la Antropología. En uno de los clásicos sobre el tema, Edmund Husserl sostuvo que las "sensaciones kinestésicas" son mecanismos no-representacionales de percepción y situación del propio cuerpo en el espacio y el tiempo. En concreto, Husserl notó que las "sensaciones kinestésicas tienen un estatus y función diferente al de los contenidos representacionales. Las kinestesias facilitan la representación, pero no [se] representan ellas mismas" (Husserl 1993: 161, traducción del autor) ${ }^{4}$. Desde esa perspectiva fenomenológica, las kinestesias se revelan en un ámbito que no coincide con el de las representaciones mentales o imaginaciones (Vorstellungen), ni con el de las representaciones mediales (Darstellungen), que son aquellas destinadas a la comunicación de contenidos (Husserl 1969: 165-167).

Trasladando esa observación desde la fenomenología al campo etnográfico, puede explorarse qué posibilidades abre la consideración del jeito quilombola y sus correspondientes kinestesias para repensar tanto las representaciones locales de la identidad política como los medios por los que ésta se comunica. Es decir: ¿Puede la atención etnográfica a las kinestesias ayudarnos a comunicarnos con el otro etnográfico -y dejar que éste nos comunique- más allá de las representaciones discursivas sugeridas por informantes selectos tales como chamanes, académicos o representantes políticos?

En mi particular socialización a lo largo del río Erepecurú, pude comprobar que, al margen de los discursos del activismo político de las élites locales, muchos de mis interlocutores apelan a la auto-conciencia de sus cuerpos, la fuerza, la destreza y el movimiento como aspectos centrales de la política, la organización sociotécnica del trabajo y la identidad colectiva. El movimiento físico no solo refleja o encarna el pasado de los grupos afroindígenas: ese movimiento también proporciona una fuente de proyecciones hacia un futuro en el cual la corporalidad juega un rol determinante (ver Arregui 2020). Desde esa perspectiva, uno puede preguntarse qué versiones del quilombolismo emergen al entender el jeito afroindígena como una "tecnología de pertenencia" que sitúa y conecta a las personas en una "ecología de prácticas" específica (Stengers 2005), pero que se desarrolla en el tiempo. En otras palabras: ¿Qué sucede al circunvalar las representaciones de la identidad etnohistórica para poner

En el original: "[Kinaesthethische Empfindungen] haben eine ganz andere Stellung und Funktion als die darstellenden Inhalte. Sie ermöglichen Darstellung, ohne selbst darzustellen" (Husserl 1993: 161). Mi interpretación coincide con otras versiones no-representacionales de las ideas de Husserl sobre el fenómeno de las kinestesias (ver Shim 2011). 
en primer plano las identificaciones con las prácticas y transformaciones de la vida cotidiana? ¿Qué proyectos ecopolíticos posibilitaría un giro de lo discursivo hacia la identidad encarnada y comunicada a través del cuerpo?

\section{Corporeizando la historia del quilombolismo}

El giro propuesto hacia lo corporal no supone la negación de la identidad histórica, sino el desplazamiento de la atención etnográfica hacia un registro cuya gestión y comunicación no esté monopolizada por élites locales y supra-locales. Precisamente, entiendo ese registro como un habitus afroindígena que refleja una "historia incorporada" (Bourdieu 1980: 111). La percepción, expresión y comunicación de esa historia requiere de una mirada etnográfica íntima, orientada por el trabajo de campo, y enfocada en aquellos movimientos que posicionan a los sujetos no tanto en la esfera político-mediática (p. ej. Planas 2014) como en el tiempo y en el espacio de las prácticas diarias desempeñadas en la selva.

Las selvas sobre las cascadas del río Erepecurú y otros tributarios del Amazonas fueron refugio para aquellos esclavos que escaparon de las plantaciones de cacao, tabaco, arroz y algodón en los siglos XVIII y XIX (Andrade 2011; De la Torre 2012; Ruiz-Peinado 2002). En ese sentido, no cabe duda de que las comunidades quilombolas del presente surgieron de aquellos primeros enclaves cimarrones. Esos asentamientos fueron más tarde reconocidos como territorios tradicionalmente ocupados por gentes que habían luchado por su derecho a una ciudadanía 'afro-descendiente' (Boaventura 2008; Almeida 2009; Boyer 2011).

Por ese motivo, la etnografía corporal aquí propuesta busca una alternativa metodológica que pueda articularse con esas luchas previas por los intereses jurídicos y territoriales de los afrodescedientes en Brasil (Arruti 2002; Boyer 2002, 2011; Larrea y Ruiz-Peinado 2004; Farfán 2015). En este momento de profunda crisis política, la reclamación de una solidaridad global pan-africanista es una de las posibles vías emergentes de reivindicación para los quilombolas (Fagundes 2020). Sin embargo, está por ver cómo esas formas de auto-representación van a oponer resistencia a algo que las políticas raciales más reaccionarias han sabido aprovechar en el caso de Brasil, a saber: el hecho de que en el "régimen multicultural de Brasil (...) el estado define a sus sujetos legítimos basándose en estándares de diferencia cultural, [aunque con ello] a menudo termina por reforzar la segregación etno-racial" de otros (Escallón 2019: 361).

Visto desde esa Amazonia que aquí llamo transparente, el poder de delimitación y comunicación etnográfica, que acaba delegado en el Estado, ha dejado en un limbo jurídico, invisibilizado, y limitado mucho las posibilidades de progreso y auto-determinación de poblaciones mestizas en las últimas décadas (Nugent y Harris 2004). Y de manera aún más alarmante, el gobierno de Jair Bolsonaro ha podido recoger esos criterios étnicos que identifican y territorializan a indígenas y quilombolas para, desde la mesa de controles de la burocracia estatal, ir desactivándolos uno a uno, o incluso ridiculizándolos en diversos actos de comunicación pública (ver Fagundes 2020).

El legado del actual mapa etnográfico de la Amazonia, producido por peritos y políticos de corte progresista en el pasado reciente, ha quedado en manos de un escéptico, un iconoclasta de las representaciones etnográficas. Ello sin duda pone de manifiesto la fragilidad de esos legados representacionales que pasan de manos de 
unas élites a otras. Resultan cruciales aquí los estudios sobre cómo la América indígena trata de reapropiarse de algunos medios tecnológicos de comunicación, como la televisión o la radio, para producir modos de auto-representación que respondan tanto al impulso modernizador como a la necesidad de resistencia a esas advenedizas racionalidades etnocéntricas o directamente racistas (ver Martínez y Orobitg 2019; Orobitg et al. 2021).

En esos gestos de resistencia, el medio de comunicación resulta, a efectos de movilización sociopolítica, tan importante como el mensaje, tal y como predica la clásica teoría de la comunicación de McLuhan (2003). En la esfera de la comunicación indígena, medios y mensajes se constituyen mutuamente y son altamente sensibles tanto a las estrategias de mediación como a los mediadores, sean éstos personas o instrumentos de comunicación. Es por ello que desde el campo académico -y en colaboración con nuestros interlocutores-cabe proponer métodos y medios de evocar y comunicar el indigenismo que no respondan únicamente a esas narraciones de un pasado y una esencia cultural. Esas narraciones no solo parecen desapegadas del día a día cambiante de las personas, sino que a menudo devienen objeto de manipulación política.

Es tal vez mediante la revelación de las condiciones materiales de la cotidianidad de las poblaciones afroindígenas donde los discursos de las élites tienen menor capacidad de tergiversación. En un estudio histórico-ecológico de la región que ocupa este ensayo, Oscar de la Torre propone la idea de "creolización ambiental" para captar, de manera empíricamente trazable, las transformaciones recíprocas de las poblaciones afro-descendientes y el entorno natural en el que se asentaron tras el cimarronaje (De la Torre 2018). Desplazando el foco desde el entorno y la historia hacia las prácticas del presente, aquí propongo observar los cuerpos también como parte constitutiva del ese paisaje llamado "Amazonia negra".

Es por ello por lo que en la sección anterior sugería que, si bien el movimiento quilombola puede ser parte de una corriente de emancipación política con años $-\mathrm{O}$ siglos- de historia, cabe considerar también la forma afroindígena de moverse como legítimo medio de expresión y comunicación de posibles "futuros" ecopolíticos (ver Arregui 2020). Aquello que localmente se identifica como el jeito, o modo de hacer, no sólo es observado en otros sino también experimentado de manera reflexiva y corporeizada. Se trata tanto de un cuerpo-lente como de un cuerpo-espejo.

Este auto-reflejo corporal no es solo político e individual, sino también histórico y colectivo. Es importante subrayar que el recurso a la kinestesia como locus del proyecto de autodeterminación afroindígena no contempla la historia que está escrita en registros, ni tampoco esa historia que puede explicarse con un argumento teleológico de unos hechos que llevaron inequívocamente a otros. La historia a la que me refiero es más bien opaca en sus representaciones, pero íntima e intensamente sentida por individuos en el seno de la colectividad.

En términos de comunicación podría decirse que, en la naturaleza de esa historia, el medio no sólo es el mensaje (McLuhan 2003), sino que el medio es más que el mensaje, es decir: el cuerpo dice o comunica más de lo que puede captar nuestro vocabulario conceptual. En la siguiente sección plantearé las posibilidades que la corporalidad como medio-mensaje ofrece al análisis de otra posible comunicación afroindígena. También señalaré las posibles limitaciones metodológicas de involucrarse etnográficamente en la comunicación de esa historia incorporada. Por un lado, argumentaré que la imbricación somática del medio, el mensaje y el mediador puede ayudar a sortear la apropiación de los medios por parte de ciertas élites, e incluso poner en cuestión la 
necesidad de representantes, ya sean políticos, culturales o intelectuales. Por otro lado, debe reconocerse que lo que llamo etnografía corporal no borra al etnógrafo como intérprete o representante, sino todo lo contrario. Por ello, describiré en primer lugar mi propia posición como etnógrafo en el campo. El gesto que propongo es el de situar con la mayor precisión y elocuencia posible la situación del espejo etnográfico, en este caso el que surge del encuentro y comparación recíproca entre distintas corporalidades 5 .

\section{Comunicación desde la (falsa) transparencia etnográfica}

Me llevó años y varias estancias de campo comprender que el espacio de las misas, celebradas en barracones de madera los días festivos, eran una plataforma de comunicación de los más diversos temas económicos, políticos, sociales o ecológicos (Figura 2). De la resistencia inicial a participar en la liturgia cristiana pasé a tomar parte de manera pasiva, sin rezar o tomar la palabra. Finalmente, unos diez años después de mis primeras visitas, y tras ser interpelado en incontables ocasiones durante las rondas de intervenciones de los feligreses, empecé a responder a aquellos comentarios que de manera indirecta o directa tematizaban mi presencia en las comunidades del río Erepecurú. Tardé diez años en asumir que mi presencia física en las comunidades, y luego también en el espacio de las misas, no podía ser gestionada como la de un observador pasivo. Fueron mis propios interlocutores los que reclamaron y corrigieron la situación del espejo etnográfico, quienes negaron mi mal pretendida transparencia.

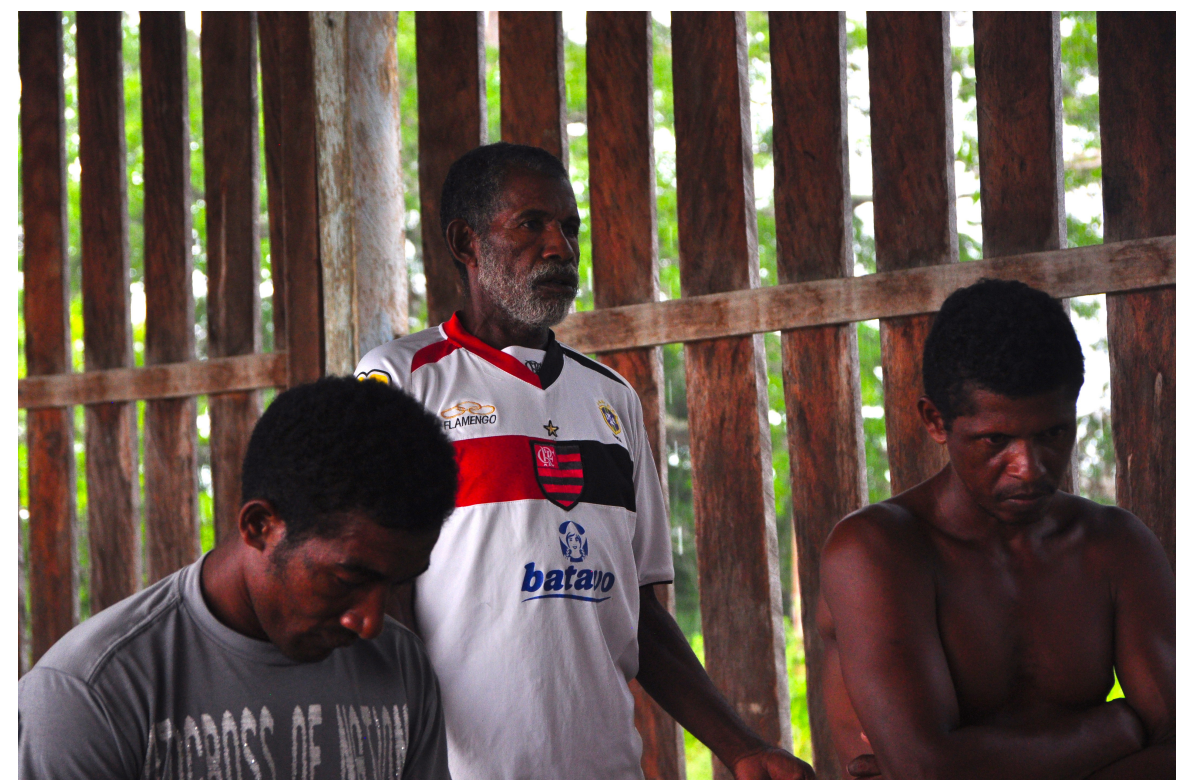

Figura 2. Los feligreses toman la palabra en las misas para discutir aspectos de la vida diaria en las comunidades. Comunidad de Varre Vento, Febrero 2019 (fotografía del autor).

Es preciso reconocer que ese cuerpo-lente/cuerpo-espejo es un medio de comunicación y diferenciación hacia el exterior al que los indígenas de América Latina han recurrido desde los primeros encuentros coloniales, tal y como recoge Eduardo Viveiros de Castro (2004: 8-9) en referencia a la interpretación Lévi-Straussiana de un célebre episodio de la Historia General y Natural de la Indias de Gonzalo Fernández de Oviedo. 
El problema de la falta de transparencia en las representaciones intelectuales fue audazmente ilustrado por Gayatri Spivak en su famoso Can the Subaltern Speak? (1988). Spivak elabora una crítica de la conversación entre Deleuze y Foucault en The Intelectuals and Power (1973), donde los dos pensadores pretenden no representar a la clase obrera con teorías intelectuales, sino centrarse sólo en los propios deseos y acciones de los subalternos (c.f. Spivak 1988: 66-69). Spivak observa que los dos pensadores franceses tratan de resistir la representación ideológica-discursiva de la clase obrera europea -un tipo representación que en el lenguaje marxista se corresponde con la idea de Darstellung (representación medial)-, pero ocluyen los deseos y acciones de una subalternidad no-europea que resulta también crucial en las estructuras de explotación del capitalismo global. Siguiendo en profundidad el vocabulario conceptual marxista que orientaba aquellos debates revolucionarios, Spivak nota que si bien Deleuze y Foucault tratan de sortear la representación teórico-discursiva de la clase obrera como Darstellung, en ese gesto incurren en una falsa transparencia de su propia posición geopolítica como intelectuales occidentales que hablan en nombre de todos los subalternos del mundo. Con ello, ejercen otra forma de representación, a la que el propio Marx denominaba Vertretung. Spivak observa que, al representarse a sí mismos como transparentes, los intelectuales tal vez resisten la representación teórica-medial o Darstellung, pero esconden su representación personal o Vertertung, es decir, su rol como representantes que hablan en nombre de otros (Spivak 1988: 70).

En una de las mencionadas misas de domingo, un hombre tomó la palabra y pronunció una larga perorata acerca de la desunión de los quilombolas de las comunidades del río Erepecurú. Con la mirada de soslayo en el extranjero allí presente, el hombre, que conocía vagamente el objeto de mi investigación, afirmó que ninguno de los insumos tecnológicos más recientes - motores fuera borda, radio- había contribuido a unir más a los quilombolas. Por el contrario, el hombre sugería que la pérdida indefectible de hábitos corporales cotidianos como remar, o trabajar la mandioca a mano, no sólo iba en detrimento de la unión, sino que ponía en cuestión la propia "identidad quilombola" (ver Arregui 2013: 164-165). En esa perorata, el hombre no erigió al etnógrafo como "representante" (Vertreter) de los quilombolas, pero incluyendo en su discurso mi objeto de investigación, contribuyó crucialmente a representar (darstellen) al antropólogo, esto es, a deshacer mi falsa transparencia en aquel contexto de comunicación.

La escena refleja el recurso propuesto por Spivak, a saber, rehuir a la representación como delegación de la palabra o Vertretung, y en su lugar mejorar los mecanismos auto-representación-Darstellung, para reflejar con nitidez - esto es, sin transparencia- la posición del intelectual-intérprete, la de los sujetos que interpreta, y las relaciones entre ambas posiciones (Spivak 1988: 84). Entiendo que es éste un gesto crucial para un proyecto decolonial en Antropología. Por ese motivo, sugiero que a un ensayo como éste, que busca dar mayor entidad a formas de ser y comunicar aparentemente diáfanas, corresponde también evocar la no-transparencia del etnógrafo en situaciones como la de las misas arriba mencionadas.

Con esta transposición etnográfica de la crítica representacional de Spivak no pretendo paralizar la etnografía en una "autoexaminacion solipsita" (Majumder 2019). Más bien, busco mostrar que las interacciones directas que acompañan al trabajo de campo estimulan ciertas representaciones del otro-etnógrafo -con su jeito inadecuado- por parte de los locales. Tal y como ejemplifica el caso presentado, es- 
tas representaciones del Otro pueden servir como útil contrapunto y visibilizador de las auto-representaciones afroindígenas.

Se trata entonces de entender el propio trabajo de campo como parte del engranaje de las representaciones y la comunicación de nuestros contextos de investigación etnográfica. En el caso que aquí nos ocupa, puede reconocerse que la aproximación al afroindigenismo basada en la evocación local de cuerpo, el movimiento y sus correspondientes kinestesias, surge de un popurrí de conceptos etnográficos y fenomenológicos de tradición intelectual claramente europea. Sin embargo, a diferencia de categorías como ancestralidad, ciudadanía étnica o tradición, las expresiones corporales kinestésicas del ser quilombola sólo son accesibles por una ruta etnográfica basada en la intimidad compartida y ajena a las representaciones preestablecidas por élites locales y supra-locales. La pregunta que quiero plantear para concluir este ensayo es si esa ruta etnográfica que subraya la centralidad ecopolítica del cuerpo, el movimiento y sus kinestesias, puede ser comunicada más allá de un reducido contexto intelectual -por ejemplo, entre antropólogas y antropólogos-, para convertirse en un proyecto de auto-representación de fuerza equiparable a la de los discursos militantes etnohistóricos sobre la Amazonia negra.

\section{Conclusiones. Auto-representación y la vida digital de los cuerpos}

Las posibilidades de auto-representación cultural y política son un aspecto central de los emergentes estudios sobre los medios de comunicación indígena (Orobitg et al. 2021). En consonancia con las ideas centrales sobre las creativas respuestas del indigenismo a la modernidad (Sahlins 1993; Turner 2007), la antropología de los medios indígenas está revelando que la apropiación de medios tecnológicos puede ser una práctica decolonial que no supone necesariamente subyugación, sino una reafirmación de la lengua, la cultura y las formas de organización indígena. Una observación central de esos estudios es que no existe un proyecto de "medios para indígenas" (Orobitg et al. 2021), sino una voluntad de participar en los medios globales. Además de una proyección hacia fuera, la exploración de los medios digitales se plantea como un espejo electrónico para la auto-observación y la reflexión sobre la identidad en un contexto global, cambiante e inter-conectado.

Una cuestión a plantear es si la propia corporalidad, en tanto que dimensión central de tantas sociedades indígenas (Breton 2006), puede formar parte de la apropiación o descolonización de los medios digitales. No debe olvidarse que el objetivo principal de los medios indígenas no es informar sino comunicar, en el sentido de establecer conexiones entre interlocutores a escalas que van de lo comunitario a lo planetario (Martínez y Orobitg 2019). En esa línea, las modernidades indígenas se han convertido en objeto de interés como lugar de reflexión sobre alternativas a nuestra historia tecno-científica (Halbmayer 2018), e incluso como modelos a través de los cuales repensar crisis globales como el cambio climático (Bold 2019). Esa entrada de lo indígena en la arena ecopolítica global no es resultado de la mediación etnográfica, sino que surge de la ya larga reivindicación de un espacio en los medios de comunicación tecnológicos e institucionales por parte de los propios indígenas (Concklin y Graham 1995; Kohn 2016).

Además del acceso a los medios de comunicación, un aspecto central para posibilitar esa urgente comunicación indígena es la traducción no sólo lingüística sino 
etnográfica, de manera que los posibles "equívocos" a propósito de cómo ordenar el mundo sean reconocidos y -en la medida de lo posible- "controlados" (Viveiros de Castro 2004). Se ha sugerido que uno de los equívocos más profundos entre el naturalismo europeo y el animismo o perspectivismo amerindio es precisamente aquel que tiene que ver con el cuerpo (Viveiros de Castro 2004; Vilaça 2005).

Sin embargo, en la última década hemos visto algunos ejemplos significativos de cómo figuras prominentes como chamanes y climatólogos recurrían a sus cuerpos en tanto que elementos de conexión entre diferentes maneras de concebir el mundo (ver Arregui 2018). Como parte de esos experimentos comunicativos, algunos actores de la ciencia y el chamanismo han tanteado los medios y registros de sus respectivos interlocutores. Podría decirse que en ese dialogo experimental cada interlocutor ha tratado de emplear los medios de comunicación del otro para hacer llegar el mensaje a otras audiencias. En esa experimentación con los medios de comunicación ajenos, el cuerpo es un tema recurrente. La referencia al cuerpo permite no solo constatar diferencias fundamentales entre ontologías o formas de definir, ordenar y relacionar lo que existe, sino que también permite trazar nuevas vías para navegar esas diferencias y comunicarse de manera efectiva. Lejos de ser un equívoco bloqueante, el cuerpo de los nuevos comunicadores puede ser proyectado incluso en los medios digitales más masivos (ver ejemplo en Nobre 2010), y convertirse en un dominio desde el que practicar un dialogo transcultural al que en otro lugar me he referido como "diplomacia ambiental" (Arregui 2019).

No obstante, si bien la vida digital de los cuerpos de esos comunicadores amplifica las posibilidades de comunicación, al mismo tiempo refuerza un problema mencionado más arriba, a saber: su rol como representantes. El papel de la antropología resulta aquí delicado. Roy Wagner identificaba la etnografía como la una "mediación universal entre voces", pero notaba también, con ironía, que a veces ejercemos el rol de "chamanes de chamanes" (Wagner 2014: 297). El peligro de entender la antropología como meta-chamanismo -especialmente la que concierne al ámbito de la Amazonia- es que al final la mediación que facilitamos entre la ciencia, el chamanismo y cualquier otro ámbito del saber, puede convertirse en un vis a vis entre élites. Para sortear el problema de la representación como Vertretung (Spivak 1988) no es suficiente con introducir el cuerpo en lo digital. También conviene eludir a los representantes habituales, y reconocer la coexistencia -y las tensiones- de las distintas versiones de la identidad corporal en un contexto determinado.

Es posible que entre esas versiones diversas y cotidianas del yo-corporal se encuentren algunas que contrasten con las representaciones de la identidad que surgen de élites chamánicas, políticas, o incluso etnográficas. Esas controversias internas del propio afroindigenismo pueden prevenirnos de una afinidad inmediata por los llamados medios digitales indígenas y lo que en ellos se comunica: una afinidad ciega que podría borrar precisamente la esencia tensional del ser indígena (Zamorano 2018).

En este ensayo he tratado precisamente de sortear a los representantes habituales del quilombolismo, y a sus proyecciones en el mundo de la comunicación digital (p.ej.: Planas 2014; Oliveira 2019). No obstante, el enfoque en lo corporal y en la vida cotidiana de las personas que encontramos en el campo tampoco garantiza una profilaxis total en relación a la representación cultural de las élites. De hecho, como John Collins ha mostrado en el contexto afro-bahíano, incluso con las perspectivas más substantivas y fieles a la cotidianidad de las poblaciones afro, la evocación de 
los hábitos diarios puede convertir la corporalidad en producto cultural, es decir, en un patrimonio alienable por parte de organismos tan poco de base como puede ser la UNESCO (Collins 2011). Es por ello que, en mi propuesta de volver la mirada sobre el cuerpo y su movimiento como medio de identificación y comunicación afroindígena, señalo la importancia de trazar y comunicar la corporalidad desde abajo y con una versión íntima de la práctica y la interacción etnográfica. Visibilizar esas identificaciones íntimas y kinestésicas es algo ciertamente difícil, pero contribuiría tal vez a mitigar el hecho de que algunos cuerpos, deseos y proyectos aparezcan apenas diluidos y con escaso margen para la auto-representación en las nuevas hegemonías de la comunicación digital.

\section{Referencias}

Almeida, Alfredo Wagner de. 2009. Tierras tradicionalmente ocupadas: Tierras de Quilombo. Tierras Indígenas. Babaçuais libres, Castañares del Pueblo, Faxinais y Fondos de Pasto. Buenos Aires: Editorial Teseo.

Andrade, Lucia. 1998. Os 300 anhos de Zumbi e os quilombos contemporâneos: Comunidades de remanescentes de quilombos. São Paulo: Comissão pro Indio - Coletânea de textos, $12-16$.

- 2011. Terras Quilombolas em Oriximiná: Pressões e Ameaças. São Paulo: Comissão Pro Índio de São Paulo.

Arregui, Aníbal G. 2013. La Selva tecnológica: sistemas sociotécnicos y Antropología simétrica en comunidades ribereñas del Bajo Amazonas. Tesis Doctoral, Universitat de Barcelona. http://hdl.handle.net/2445/35241.

—. 2014. «"Teoría", "inteligencia” y "ciencia” como índices bajo amazónicos del conocimiento incorporado». Amazônica. Revista de Antropologia 6 (1): 90-108

—. 2015. «Amazonian Quilombolas and the Technopolitics of Aluminum». Journal of Material Culture 20 (3): 149-172.

- 2018. «Ribeirinho Hunting Techno-Animism. On the Inexact Lines of Amazonian Modernity», en Indigenous Modernities in South America, Ernst Halbmayer, ed., pp. 164183. Herefordshire: Sean Kingston Publishing.

—. 2019. «This Mess is a 'World'! Environmental Diplomats in the Mud of Anthropology», en Indigenous Perceptions of the End of the World. Creating a Cosmopolitics of Change, Rosalyn Bold, ed., pp. 183-202. Palgrave Studies in Anthropology of Sustainability. Londres: Palgrave Macmillan.

-. 2020. «The Quilombola Movement. Sensing Futures in Afroindigenous Amazonia». Ethos 48: en prensa.

Arruti, José Mauricio. 2002. De como a cultura se faz política e vice-versa: sobre religiões, festas, negritudes e indignidades no Nordeste contemporâneo». Texto presentado en "IV Ciclo Nação e Região Brasil 500 anos Experiência e Destino". Río de Janeiro: Fundação Nacional de Artes, Universidade do Estado do Rio de Janeiro, Universidade Estadual do Norte Fluminense.

Barbosa Livia, de H. 1995. «The Brazilian Jeitinho: An Exercise in National Identity», en The Brazilian Puzzle: Culture on the Borderland of the Western World, David Hess y Roberto DaMatta, eds., pp. 35-48. Nueva York: Columbia University Press.

Bloch, Maurice E. F. 1998. How We Think They Think: Anthropological Approaches on Cognition, Memory and Literacy. Boulder: Westview Press. 
Boaventura Leite, Ilka. 2008. «O projeto político quilombola: Desafios, conquistas e impasses atuais». Revista de Estudos Feministas 16 (3): 965-977.

Bold, Rosalyn, ed. 2019. Indigenous Perceptions of the End of the World. Creating a Cosmopolitics of Change. Palgrave Studies in Anthropology of Sustainability. Londres: Palgrave Macmillan.

Bourdieu, Pierre.1980. Le sens pratique. París: Éditions Minuit.

Boyer, Véronique. 2002. «Quilombolas et Évangéliques: Une Incompatibilité Identitaire? Réflexions à partir d'une étude de cas en Amazonie Brésilienne». Journal de la Société des Américanistes 88: 159-178.

—. 2011. «Os quilombolas no Brasil: ¿Pesquisa antropológica ou perícia político-legal?». Nuevo Mundo Mundos Nuevos, Cuestiones del tiempo presente. https://journals.openedition.org/nuevomundo/61721

Breton, Stéphane, dir. 2006. Qu'est-ce qu'un corps? Afrique de l'Ouest, Europe, occidentale, Nouvelle-Guinée, Amazonie. París: Flammarion, Musée du quai Branly.

Collins, John. 2011. «Melted Gold and National Bodies: the Hermeneutics of Depth and the Value of History in Brazilian Racial Politics». American Ethnologist 38 (4): 683-700.

Concklin, Beth A. y Laura Graham. 1995. «The Shifting Middle Ground: Amazonian Indians and Eco-Politics». American Anthropologist New Series 97 (4): 695-710.

De la Torre, Oscar. 2012. "'The Land is Ours and We Are Free to Do All that We Want': Quilombos and Black Rural Protest in Amazonia, Brazil, 1917-1929». The Latin Americanist 56: 33-56.

-. 2018. The People of the River: Nature and Identity in Black Amazonia, 1835-1945. Chapel Hill: University of North Carolina Press.

Downey, Greg. 2010. "'Practice without Theory’: A Neuroanthropological Perspective in Embodied Learning». Journal of the Royal Anthropological Institute 16: S22-S40.

Escallón, Maria. 2019. «Rights, Inequality, and Afro-Descendant Heritage in Brazil». Cultural Anthropology 34 (3): 359-387.

Fagundes, Guilherme Moura. 2020. «Reclaiming Quilombismo in the End of the Conciliations». Cultural Anthropology, Hot Spots, Fieldsights, January 28. https://culanth.org/fieldsights/reclaiming-quilombismo-in-the-end-of-the-conciliations.

Farfán Santos, Elisabeth. 2015. «"Fraudulent” Identities: The Politics of Defining Quilombo Descendants in Brazil». The Journal of Latin American and Caribbean Anthropology 20 (1): 110-132. https://doi.org/10.1111/jlca.12108.

Foucault, Michel y Gilles Deleuze. 1973. «The Intellectuals and Power: A Discussion between Gilles Deleuze and Michel Foucault». Telos: Critical Theory of the Contemporary 16: 103-109.

Goldman, Marcio. 2014. «A relação afroindígena». Cadernos De Campo 23: 213-222. https://doi.org/10.11606/issn.2316-9133.v23i23p213-222.

González-Abrisketa, Olatz y Carlos García-Grados. 2018. «iIncorporar, encarnar, encorporar y/o corporear? Un ejercicio de traducción para repensar la agentividad de los cuerpos», en Etnografias feministas. Una mirada al siglo XXI desde la antropología vasca, Mari Luz Esteban y José M. Hernández García, coords., pp. 231-249. Bellaterra: Edicions Bellaterra.

Halbmayer, Ernst, ed. 2018. Indigenous Modernities in South America. Herefordshire: Sean Kingston Publishing.

Harris, Mark. 2005. «Riding a Wave: Embodied Skills and Colonial History on the Amazon Floodplain» Ethnos: Journal of Anthropology 70 (2): 197-219.

Husserl, Edmund. 1969. Die Krisis der europäischen Wissenschaften und die transzendentale Phänomenologie. The Hage: Martinus Nijhof. 
—. 1993 (1907). Ding und Raum. Vorlesungen 1907. Hamburgo: Felix Meiner Verlag.

Kohn, Eduardo. 2016. «Ecopolitics» Theorizing the Contemporary, Fieldsights, January 21. https://culanth.org/fieldsights/ecopolitics.

Larrea, Christina y Luis Ruiz-Peinado. 2004. «Memoria y territorio quilombola en Brasil». Quaderns de l'Institut Català d'Antropologia 20: 191-215.

Majumder, Atreyee. 2018. «Fieldwork and the Native Informant: A Review of Culture at Large 2017». Member Voices, Fieldsights, January 23. https://culanth.org/fieldsights/ fieldwork-and-the-native-informant-a-review-of-culture-at-large-2017

Martínez Mauri, Mònica y Gemma Orobitg. 2019. «Internet fortalece a las comunidades indígenas». The Conversation. https://theconversation.com/internet-fortalece-a-las-comunidades-indigenas-110251.

Mauss, Marcel.1968. Les techniques du corps. Sociologie et anthropologie. París: Presses Universitaires de France.

Mcluhan, Marshall. 2003. Understanding Media. The Extensions of Man. Berkley: Gingko Press.

Moran, Dermot y Joseph Cohen. 2012. The Husserl Dictionary. Londres y Nueva York: Continuum.

Nobre, Antonio D. 2010. «The Magic of the Amazon: A River that Flows Invisibility all around us». TED talk. https://www.ted.com/talks/antonio_donato_nobre_the_magic_of_ the_amazon_a_river_that_flows_invisibly_all_around_us/

Nugent, Steven y Mark $\bar{H}$ arris, eds. 2004 . Some Other Amazonians: Perspectives on Modern Amazonia. Londres: Institute for the Study of Americas.

Oliveira, Joana. 2019. «A Amazônia também é negra». El País, 20 Noviembre. https://brasil. elpais.com/brasil/2019/11/19/politica/1574164761_425337.html.

Orobitg, Gemma y Roger Canals. 2020. «Hipertexto, multivocalidad y multimodalidad para una etnografía sobre los medios de comunicación: la web MEDIOS INDÍGENAS». Revista Española de Antropología Americana 50: 215-227.

Orobitg, Gemma, Gemma Celigueta, Francisco M. Gil García, Julián López García, Mònica Martínez Mauri, Óscar Muñoz Morán, Roger Canals, Gabriel Izard, Sebastián Gómez Ruiz, Beatriz Pérez Galán, Pedro Pitarch. 2021. «Los medios indígenas en América Latina. Una práctica descolonizadora». Revista de Historia (Universidad Nacional de Costa Rica) 83 (en prensa).

Pfaffenberger, Bryan. 1992. «Social Anthropology of Technology». Annual Review of Anthropology 21: 491-516.

Planas, Roque. 2014. «Brazil's “Quilombo” Movement May Be the World's Largest Slavery Reparations Program». The Huffington Post, 7 Octubre. https://tinyurl.com/yxl4js18.

Ruiz-Peinado, Luis. 2002. Cimarronaje en el Brasil: Mocambos del Trombetas. Vilanova i la Geltrú: Edicions Cep i Nansa.

Sahlins, Marshal. 1993. "Goodbye to Tristes Tropes: Ethnography in the Context of Modern World History». Journal of Modern History 65 (1): 1-25

Sauma, Julia. 2014. «Entrosar-se, uma reflexão etnográfica afroindígena». Cadernos De Campo 23: 257-270. https://doi.org/10.11606/issn.2316-9133.v23i23p257-270.

Scaramuzzi, Igor. 2019. «Arte de "arremedar": atos de criação entre humanos e animais no Alto Trombetas (Pará, Brasil)». Etnográfica 23 (1): 69-86.

Shim, Michael. K. 2011. «Representationalism and Husserlian Phenomenology». Husserl Studies 27 (3): 197-215.

Spivak, Gayatri Chakravorty. 1988. «Can the Subaltern Speak?», en Marxism and the Interpretation of Culture, Cary Nelson y Lawrence Grossberg, eds., pp. 271-313. Urbana: University of Illinois Press. 
Stengers, Isabelle. 2005. «Introductory Notes to an Ecology of Practices». Cultural Studies Review 11 (1): 183-196.

Turner, Terence S. 2007. «Indigenous Resurgence, Anthropological Theory, and the Cunning of History». Focaal-European Journal of Anthropology 49: 118-123.

Vilaça, Aparecida. 2005. «Chronically Unstable Bodies: Reflections on Amazonian Corporalities». Journal of the Royal Anthropological Institute 11 (3): 445-464.

Viveiros de Castro, Eduardo. 2004. «Perspectival Anthropology and the Method of Controlled Equivocation». Tipiti: Journal of the Society of Lowland South-America 2 (1): $3-22$.

Wagner, Roy. 2014. «The Rising Ground». HAU: Journal of Ethnographic Theory 4 (2): 297-300.

Zamorano Villarreal, Gabriela. 2018. «The Risks of Affinity: Indigenity and Indigenous Film Production in Bolivia», en Who Are 'We'?: Reimagining Alterity and Affinity in Anthropology, Liana Chua y Nayanika Mathur, eds., pp. 128-150. Nueva York, Oxford: Berghahn Books. 